\title{
X-ray Morphology of Clusters of Galaxies
}

\author{
H. Böhringer, R.A. Schwarz, U.G. Briel, H. Ebeling, W. Voges \\ Max-Planck-Institut für Extraterrestrische Physik \\ 8046 Garching, Germany
}

\begin{abstract}
The ROSAT observatory with its high spatial resolution $\mathrm{X}$-ray telescope is an ideal instrument for the study of clusters of galaxies. In the first part of the mission an All Sky X-ray Survey was conducted with ROSAT. Here we present first results of observations of Virgo, Perseus and some other clusters of galaxies from the All Sky Survey. These data illustrate the capabilities of the ROSAT observatory for studies of the morphological structure and physical properties of galaxy clusters.
\end{abstract}

\section{Introduction}

Clusters of galaxies constitute a major fraction among the brightest extragalactic X-ray sources. The X-ray emission originates from a hot, optically thin intracluster plasma that typically has temperatures between 2 and $10 \mathrm{keV}$. The soft X-ray energy window of the ROSAT X-ray telescope ( 0.1 to $2.4 \mathrm{keV}$ ) covers part of the wavelength region where the radiative emission of the intracluster medium has its maximum. The ROSAT X-ray telescope (e.g. Trümper et al., 1991) with its good spatial resolution is therefore ideally suited for morphological studies of clusters of galaxies.

The two focal plane instruments, the Position Sensitive Proportional Counter (PSPC) and the High Resolution Imager (HRI), have a spatial resolution better than 30 and 5 arcsec near the telescope axis, respectively. The spectral resolution (of about $45 \%$ at $1 \mathrm{keV}$ ) and the good calibration of the PSPC detector allow to determine the temperature of hot, thermal, X-ray emitting plasma for temperatures below $1 \mathrm{keV}$ where the cutoff in the free-free radiation spectrum falls into the ROSAT energy window. For temperatures above $1 \mathrm{keV}$, which are more relevant for observations of clusters of galaxies, the change of the spectral shape in the ROSAT window is quite small so that temperatures can only roughly be determined for good photon statistics. On the other hand the emissivity in the ROSAT band has very little dependence on temperature, so that gas densities and gas masses can well be determined without precise temperature information.

The major advances in the investigation of clusters of galaxies with the ROSAT observatory will therefore lay in a better determination of the mass and distribution of the cluster gas as well as its energy budget, in a better estimate of the gravitating mass of the cluster within the uncertainties of the temperature information, and in the study of the cluster morphology and thus its dynamical state. The following examples will illustrate the capabilities of the ROSAT X-ray telescope. In the calculations a Hubble constant of $H_{0}=50 \mathrm{~km} \mathrm{Mpc}^{-1} \mathrm{~s}^{-1}$ was adopted. 


\section{The Virgo Cluster}

The Virgo cluster covers an area in the sky with more than 10 degress diameter. Therefore it has never been imaged with an X-ray telescope as a whole. Scans of the cluster region by the collimated detector of GINGA indicated extended X-ray emission on scales of several degrees. In the ROSAT All Sky Survey the Virgo cluster region was scanned with an average exposure time of about 460 sec. Fig. 1 shows a contour plot of the Survey data. For this plot only the hard photons $(E \geq 0.4$ $\mathrm{keV}$ ) have been used to enhance the signal to noise ratio and the image has been smoothed with a Gaussian filter. The most prominent features are the giant X-ray halo of M87 and the much smaller halo of M49 (NGC 4772). Besides the almost spherically symmetric halos there is also diffuse emission between the two halos that is clearly observed for the first time. The assymmetrical extent to the east between the two halos, is also visible in the contour plots of the galaxy densities from the optical survey by Bingeli et al. (1987).

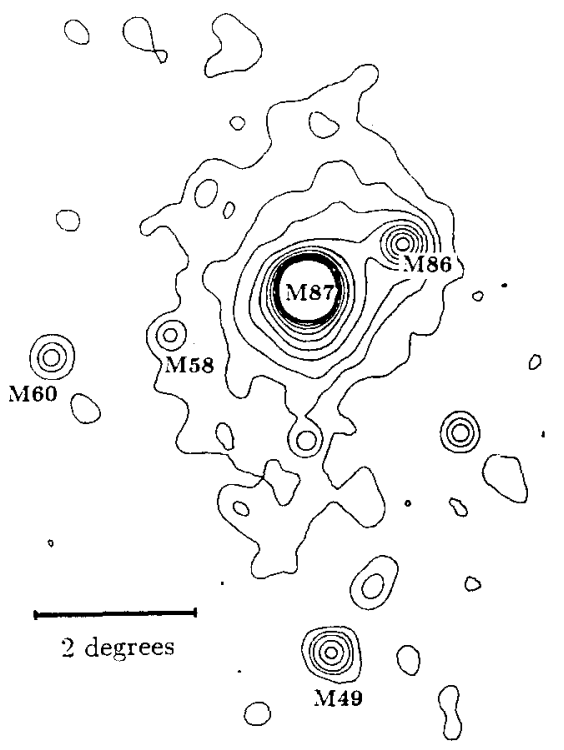

Fig. $1 \mathrm{X}$-ray image of the Virgo cluster of galaxies from the ROSAT All Sky Survey. In this contour plot only photons with an energy $\geq 0.4 \mathrm{keV}$ where included and the image has been smoothed by a Gaussian filter with a width of 20 arcmin. The most prominent elliptical galaxies with observed $\mathrm{X}$-ray emission are labled in the plot.

Most of the emission comes from a halo around M87 which is almost spherically symmetric at least out to a radius of two degrees. This region contains more than 25,000 photons in the ROSAT Survey, enough for a very detailed spectral analysis. A $\chi^{2}$ fit of model spectra to the ROSAT data for this region gives a temperature of $2.4\left[\begin{array}{c}+0.35 \\ -0.2\end{array}\right] \mathrm{keV}$ which is in good agreement with the results of the GINGA satellite by Koyama et al. (1991) who found a temperature of $2.19 \pm 0.04) \mathrm{keV}$. The rise in the cluster gas temperature to the south of M87 which was observed by GINGA (Koyama et al, 1991) is not confirmed by the ROSAT data, however. If the temperature determination is performed for different concentric rings in the halo of M87 a significant temperature drop is observed in the innermost part of the cluster, at radii $\leq 15$ arcmin. Fig. 2 shows the temperature profile obtained for a binning with boundaries at $7,15,30$, and 60 arcmin.

A deprojection analysis of the surface brightness profile (following Fabian et al, 1981) yields a 
cooling flow radius of about $120 \mathrm{kpc}$ inside which the cooling time is shorter than the Hubble time. This cooling flow radius of $\sim 20$ arcmin is consistent with the observed temperature drop inside a 15 arcmin radius and also the magnitude of the temperature decrease is within the limits of the predictions of a one-phase cooling flow model. The mass deposition rate in the cooling flow was found to have a value of $20( \pm 4) \mathrm{M}_{\odot} \mathrm{y}^{-1}$ in good accord with the results by Stewart et al.(1984).

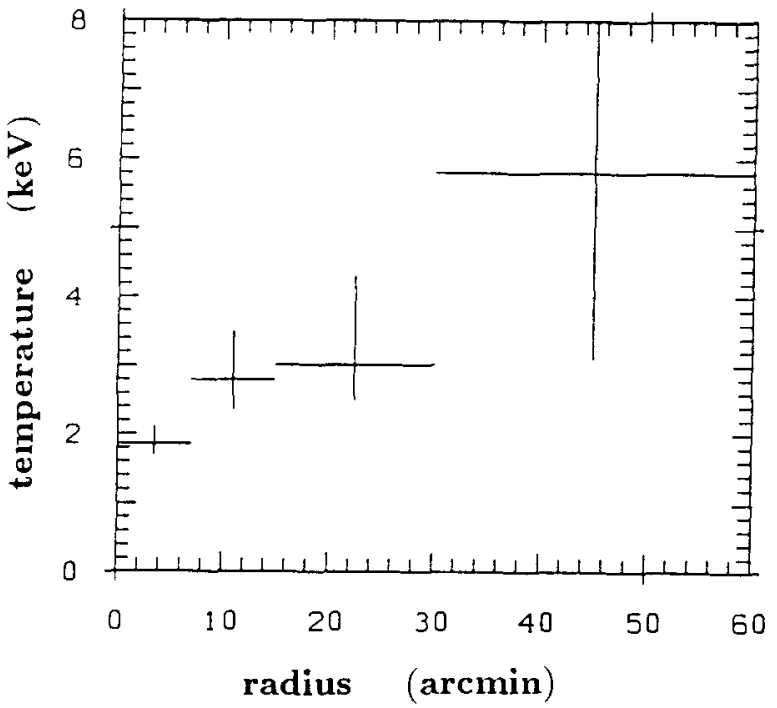

Fig. 2 Temperature profile of the gas in the X-ray halo of M87. The vertical error bars show the $2 \sigma$ uncertainties.

\section{The Perseus Cluster}

The brightest cluster in the Survey is the Perseus cluster of galaxies. Fig. 3 shows an image of Perseus from the All Sky Survey which again covers an area larger than explored by previous X-ray telescopes (Schwarz et al., 1991). For the first time a trace of the most prominent optical feature in Perseus, the bright chain of galaxies from NGC 1275 to IC 310 , can clearly be seen in the X-ray image. The total ROSAT PSPC count rate of Perseus within a radius of $42 \operatorname{arcmin}$ is $33.6 \mathrm{cts} \mathrm{s}$ corresponding to a luminosity of $1.4 \cdot 10^{45} \mathrm{erg} \mathrm{s}^{-1}$.

The mass profile of the X-ray emitting gas can be determined from a deprojection of the surface brightness profile (assuming spherical symmetry). A radius of $2 \mathrm{Mpc}$ encloses a gas mass of about $2 \cdot 10^{14} \mathrm{M}_{\odot}$. From optical data one estmiates a virial mass of about $16 \cdot 10^{14} \mathrm{M}_{\odot}$ (Kent and Sargent, 1983), while the analysis of recent X-ray observations with some extrapolation yield a total mass of the order of $7 \cdot 10^{14} \mathrm{M}_{\odot}$ (Eyles et al, 1991). The ROSAT results show that the fraction of the mass contained in the hot gas is very high (12-29\% in the case of Perseus) out to radii as large as $2 \mathrm{Mpc}$.

An analysis of the X-ray spectra in concentric rings around the central galaxy NGC 1275 shows a significant temperature drop in the cooling flow region like in the case of M87. While the overall temperature of the cluster can be determined as $5.3( \pm 1.3) \mathrm{keV}$, the temperature minimum is between 2 and $3 \mathrm{keV}$.

There is a long standing problem in the interpretation of Perseus observations that the virial mass estimate from galaxy positions and redshifts is more than a factor of 2 higher than the mass distribution consistent with models for the X-ray surface brightness and temperature (Kent and 
Sargent, 1983; Fabian et al., 1981; Eyles et al., 1991). A careful analysis of the ROSAT X-ray data show that there is a large scale temperature anisotropy in Perseus outside the cooling flow region (Schwarz et al., 1991). The temperature in the east is significantly lower ( 2 to $3.5 \mathrm{keV}$ ) than the western side. This may imply that Perseus suffered a very recent infall of a larger subcomponen which would explain the discrepancy in the mass estimates.

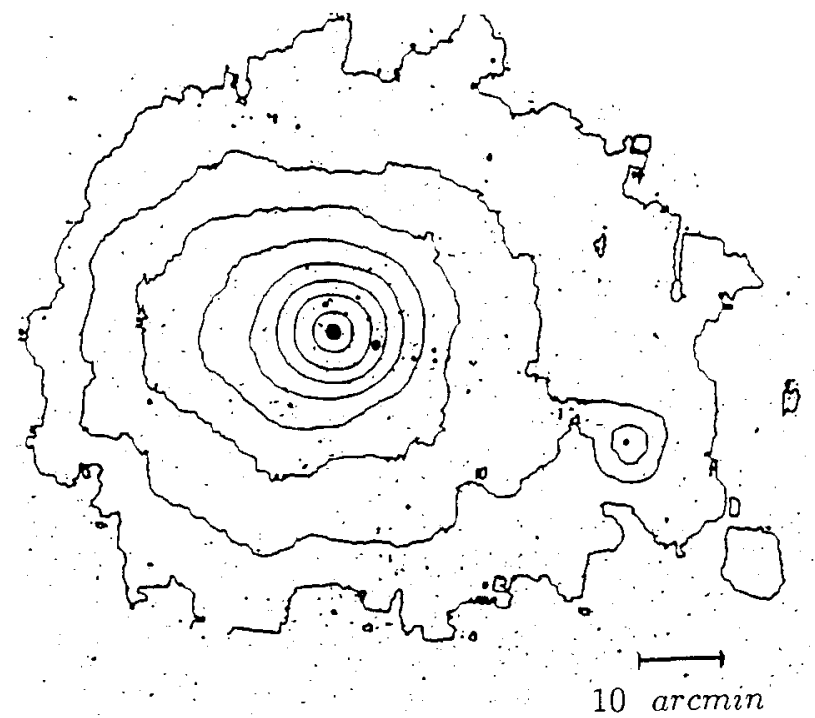

Fig. $3 \mathrm{X}$-ray image of the Perseus cluster from the All Sky Survey. The contour levels are logarithmically spaced. The $\mathrm{X}$-ray map is superposed onto the POSS plate. The main amximum coincides with NGC 1275 while the small western maximum is at the position of IC 310 .

\section{Results from other cluster observations}

The ROSAT observations have revealed very interesting substructure also in other clusters of galaxies. In the Survey image of the Coma cluster a large second component was discovered to the south west of the main cluster (Briel et al, 1991b). This second part has a lower temperature than the main component. In an early pointed observation of A2256 the cluster was found to be a superposition of two cluster components which are in the process of merging (Briel et al., 1991a). This double component structure is also reflected in the optical data and provides a nice explanation for the very complex morphology of the radio halo in A2256. Similar structural details are now being found in the analysis of other clusters in the ROSAT Survey. If one could obtain a quantitative measure for the occurance of substructure from these X-ray observations one could possibly set a lower limit on the evolution of clusters at present and on the mean density of the Universe.

In the first processing of the ROSAT All Sky Survey of the order of 50,000 X-ray sources have been detected. We expect to find more than 4000 clusters of galaxies among these sources. About 1000 clusters from the Abell, Corwin and Olowin catalogue (ACO, 1989) were readily identified and a smaller number of clusters from other catalogues were also found. Two thirds of the expected number of clusters should be new detections and have to be varified by further optical identification (correlation with material from optical plates and by observations). In reverse only about 16 to $20 \%$ of the clusters listed in the ACO catalogue are found in the ROSAT survey. Thus the final list of $\mathrm{X}$-ray selected clusters will be quite different from the present optical catalogues. Redshift surveys on ROSAT clusters of galaxies have already been started and the nature of more than 20 newly detected clusters has been confirmed by the measurement of several coincident reshifts per cluster. 


\section{Summary}

The results from ROSAT observations of individual clusters of galaxies have shown that far more details in the cluster structures can be resolved, using the higher spatial resolution and the energy discrimination of the ROSAT PSPC detector, than possible up to now. The few ROSAT HRI images which have been analysed so far promise even higher spatial resolution. In particular the temperature variation observed in cooling flow clusters confirms the previous interpretation of the cluster surface brightness profile by cooling flow models. Alternative models where the radiative losses are balanced by heating have to be extremely fine tuned to reproduce the observed temperature distribution. It was also found that X-ray imaging is a very powerfull tool to study the substructure and the dynamical state of clusters. The ROSAT observations of Perseus and A 2256 for example show more details than can be obtained with a sample of serveral hundred galaxy redshifts. While the statistical quality of optical observations is limited by the number of observable galaxies, the quality of X-ray observations is determined by the exposure time. The program of long pointed observations of clusters, part completed and part still in planning, promise a far more detailed determination of cluster masses, gas content, and dynamical states of clusters.

An extremely interesting result from the ROSAT All Sky Survey is the fact that probably only one third of the X-ray detected clusters of galaxies are known optically and only a fraction of the known optical clusters are found in the Survey. Surveying in X-rays one is more sensitive for the deepest parts of the potential wells of clusters while the optical detection depends more on the global morphology. It is interesting that both detection techniques lead to quite different cluster samples. The X-ray selected all sky sample of clusters of galaxies will therefore be very important for cosmological studies such as a spatial correlation analysis, analysis of the cluster mass function, and evolution studies.

Acknowledgements We would like to thank the ROSAT team for the help in the data analysis and for making these unique observations possible. H.B. acknowledges support from the Deutsche Forschungsgemeinschaft.

\section{References}

Abell, G.O., Corwin, H.G., Olowin, R.P., 1989, Astrophys. J. Suppl, 70, 1.

Binggeli, B., Tammann, G.A., Sandage, A., 1987, Astron. J., 94, 251.

Briel, U.G., Henry, J.P., Schwarz, R.A., Böhringer, H., Ebeling, H., Edge, A.C., Hartner, G.D., Schindler, S., \& Voges, W., 1991a, Astron. Astrophys., 246 L10.

Briel, U.G., Henry, and Böhringer, H., 1991b, in Clusters and Superclusters of Galaxies, Colles, M.M., Babul, A., Edge, A.C., Johnstone, R.M., and Raychaudhury (eds.), Institute of Astronomy, Cambridge, p91.

Eyles, C.J., Watt, M.P., Bertram, D., Church, M.J., Ponman, T.J., Skinner, G.K., Willmore, A.P., 1991, Astrophys. J., 376, 23.

Fabian, A.C., Hu, E.M., Cowie, L.L., Grindley, J., 1981, Astrophys. J., 248, 47.

Kent, S.M., and Sarget, W.L.W., 1983, Astron. J., 88, 697.

Koyama, K., Takano, S., and Tawara, Y., 1991, Nature, 350, 135.

Schwarz, R., Edge, A., Voges, W., Böhringer, H., Ebeling, H., Schartel, N., and Briel, U., 1991, in Clusters and Superclusters of Galaxies, Colles, M.M., Babul, A., Edge, A.C., Johnstone, R.M., and Raychaudhury (eds.), Institute of Astronomy, Cambridge, p43.

Stewart, G.C., Canizares, C.R., Fabian, A.C., Nulsen, P.E.J., 1984, Astrophys. J., 278, 536.

Trümper, J., et al., 1991, Astron. Astrophys., 246 L1. 